Advances in Dynamical Systems and Applications (ADSA).

ISSN 0973-5321, Volume 15, Number 1, (2020). 73-82

(C) Research India Publications

https://dx.doi.org/10.37622/ADSA/15.1.2020.73-82

\title{
Coupled Fixed Point Theorem for Mapping Satisfying Integral Type Contraction on Cone Metric Space
}

\author{
Surendra Kumar Tiwari ${ }^{1}$, Ayushi Saxena ${ }^{2}$ and Shantanu Bhaumik *3 \\ ${ }^{1,2,3}$ Department of Mathematics, Dr. C. V. Raman University, Bilaspur (C.G.), India.
}

\begin{abstract}
In this paper, the existence and uniqueness of coupled fixed point theorem for mapping satisfying integral type contraction on cone metric space has been proved. The result is verified with the help of suitable example.
\end{abstract}

Mathematics Subject Classification (2010): 47H09, 47H10, 49J25.

Keywords: Coupled fixed point, Integral type contraction, Cone metric space, Fixed point.

\section{INTRODUCTION AND MATHEMATICAL PRELIMINARIES}

In 2002, Branciari [13] gave a modified version of the Banach contraction principle which is mainly integral type inequality for a single mapping and showed the following memorable fixed point theorem.

Theorem 1. Let $(X, d)$ be a complete metric space, $a \in(0,1)$, and let $f: X \rightarrow X$ be a mapping such that for each $x, y \in X$,

$$
\int_{0}^{d(f x, f y)} \varphi(t) d t \leq a \int_{0}^{d(x, y)} \varphi(t) d t,
$$

where $\varphi:[0,+\infty) \rightarrow[0,+\infty)$ is a Lebesgue-integrable mapping which is summable on each compact subset of $[0,+\infty)$, nonnegative, and such that

$$
\forall \epsilon>0, \quad \int_{0}^{\epsilon} \varphi(t) d t>0 .
$$

Then, $f$ admits a unique fixed point $p \in X$ such that for each $x \in X, \lim _{n \rightarrow \infty} f^{n} x=p$. Theorem 1 is a generalization of the Banach-Caccioppoli [8] principle.

${ }^{*}$ Corresponding Author 
In 2006, The concept of coupled fixed point was given by Bhaskar and Lakshmikantham in [2]. After that several authors proved various coupled and common coupled fixed point theorem in partial ordered metric space, $G$-metric space, $b$-metric space, fuzzy metric space, cone metric space etc. Some of the works are noted in [4], [5], [9].

In 2007, Huang and Zhang [1] introduced the concept of cone metric space, where they generalized metric space by replacing the set of real numbers with an ordering Banach space. Thus, the cone naturally induces a partial order in Banach space. Some of the works are noted in [6], [7], [10], [11].

The aim of this paper is to establish the existence and uniqueness of coupled fixed point theorem satisfying integral type contraction in cone metric space. In this paper we do not impose the normality condition of the cone. The only assumption is that cone $\mathrm{P}$ has nonempty interior.

\section{Some definitions and results are as follows:}

Definition 2. [1] Let $E$ be a real Banach space and $P$ be a subset of $E$. $P$ is called a cone if and only if:

(i) $P$ is nonempty, closed, and $P \neq\{0\}$;

(ii) $a, b \in \mathbb{R}, \quad a, b \geq 0 ; \quad x, y \in P \Longrightarrow a x+b y \in P$;

(iii) $x \in P$ and $-x \in P \Longrightarrow x=0$;

Given a cone $P \subset E$, a partial ordering $\leq$ with respect to $P$ is naturally defined by $x \leq y$ if and only if $x-y \in P$ for $x, y \in E$. We shall write $x<y$ to indicate that $x \leq y$ but $x \neq y$, while $x \ll y$ will stand for $y-x \in \operatorname{int} P$, where int $P$ denote the interior of $P$.

The cone $P$ is said to be normal if there exists a real number $K>0$ such that for all $x, y \in E$,

$$
0 \leq x \leq y \longrightarrow\|x\| \leq K\|y\| .
$$

The least positive number $K$ satisfying the above statement is called the normal constant of $P$.

The cone $P$ is called regular if every increasing sequence which is bounded from above is convergent; that is, if $\left\{x_{n}\right\}$ is a sequence such that

$$
x_{1} \leq x_{2} \leq \cdots \leq x_{n} \leq \cdots \leq y,
$$

for some $y \in E$, then there is $x \in E$ such that $\left\|x_{n}-x\right\| \longrightarrow 0$ as $n \longrightarrow \infty$. Equivalently, the cone $P$ is regular if and only if every decreasing sequence which is bounded from below is convergent. It is well known that a regular cone is a normal cone. 
In the following we always suppose that $E$ is a real Banach space with cone $P$ in $E$ with int $P \neq \emptyset$ and $\leq$ is partial ordering with respect to $P$.

Definition 3. [1] Let $X$ be a nonempty set. Let the mapping $d: X \times X \longrightarrow E$ satisfies:

(i) $0 \leq d(x, y)$, for all $x, y \in X$ and $d(x, y)=0$ if and only if $x=y$;

(ii) $d(x, y)=d(y, x)$, for all $x, y \in X$;

(iii) $d(x, y) \leq d(x, z)+d(z, y)$, for all $x, y, z \in X$.

Then $d$ is called a cone metric on $X$ and $(X, d)$ is called a cone metric space.

Definition 4. [1] Let $(X, d)$ be a cone metric space, $\left\{x_{n}\right\}$ a sequence in $X$ and $x \in X$.

(i) If for every $c \in E$ with $0 \ll c$, there exists $n_{0} \in \mathbb{N}$ such that for all $n>n_{0}, \quad d\left(x_{n}, x\right) \ll c$, then $\left\{x_{n}\right\}$ is said to be convergent and $\left\{x_{n}\right\}$ converges to $x$, and $x$ is the limit of $\left\{x_{n}\right\}$. This limit is denoted by $\lim _{n} x_{n}=x$ or $x_{n} \longrightarrow x$ as $n \longrightarrow \infty$.

(ii) If for every $c \in E$ with $0 \ll c$, there exists $n_{0} \in N$ such that for all $n, m>n_{0}, \quad d\left(x_{n}, x_{m}\right) \ll c$, then $\left\{x_{n}\right\}$ is called a Cauchy sequence in $X$.

(iii) If every Cauchy sequence in $X$ is convergent in $X$, then $X$ is called a complete cone metric space.

Lemma 5. [6] If $E$ be a real Banach space with cone $P$ in $E$, then for $a, b, c \in E$,

(i) If $a \leq b$ and $b \ll c$, then $a \ll c$.

(ii) If $a \ll b$ and $b \ll c$, then $a \ll c$.

Definition 6. [2] An element $(x, y) \in X \times X$ is called a coupled fixed point of the mapping $F: X \times X \longrightarrow X$ if $x=F(x, y)$ and $y=F(y, x)$.

\section{MAIN RESULTS}

Theorem 7. Let $(X, d)$ be a complete cone metric space with a cone $P$ having nonempty interior. Let $F: X \times X \longrightarrow X$ satisfying

$$
\begin{aligned}
\int_{0}^{d(F(x, y), F(u, v))} \phi(t) d t & \leq \alpha \int_{0}^{d(x, u)} \phi(t) d t+\beta \int_{0}^{d(y, v)} \phi(t) d t+\gamma \int_{0}^{d(u, F(x, y))} \phi(t) d t \\
& +\delta \int_{0}^{d(u, F(u, v))} \phi(t) d t
\end{aligned}
$$

for all $x, y, u, v \in X$, and $\alpha, \beta, \gamma, \delta \in(0,1)$ such that $\alpha+\beta+\gamma+\delta<1$. and $\phi(t): P \rightarrow P$ is a Lebesgue-integral mapping which is summable, nonnegative and 
such that for all $0 \ll \epsilon, 0 \ll \int_{0}^{\epsilon} \phi(t) d t$.

Then $F(x, y)$ has a unique coupled fixed point in $X \times X$.

Proof. Let $x_{0}$ and $y_{0}$ be two arbitrary points in X.

Let

$$
\begin{array}{cc}
x_{2 k+1}=F\left(x_{2 k}, y_{2 k}\right), & y_{2 k+1}=F\left(y_{2 k}, x_{2 k}\right) . \\
x_{2 k+2}=F\left(x_{2 k+1}, y_{2 k+1}\right), & y_{2 k+2}=F\left(y_{2 k+1}, x_{2 k+1}\right) .
\end{array}
$$

for $\mathrm{k}=0,1,2,3, \ldots$ Then

$$
\begin{aligned}
\int_{0}^{d\left(x_{2 k+1}, x_{2 k+2}\right)} \phi(t) d t & =\int_{0}^{d\left(F\left(x_{2 k}, y_{2 k}\right), F\left(x_{2 k+1}, y_{2 k+1}\right)\right)} \phi(t) d t \\
& \leq \alpha \int_{0}^{d\left(x_{2 k}, x_{2 k+1}\right)} \phi(t) d t+\beta \int_{0}^{d\left(y_{2 k}, y_{2 k+1}\right)} \phi(t) d t \\
& +\gamma \int_{0}^{d\left(x_{2 k+1}, F\left(x_{2 k}, y_{2 k}\right)\right)} \phi(t) d t+\delta \int_{0}^{d\left(x_{2 k+1}, F\left(x_{2 k+1}, y_{2 k+1}\right)\right)} \phi(t) d t \\
& \leq \alpha \int_{0}^{d\left(x_{2 k}, x_{2 k+1}\right)} \phi(t) d t+\beta \int_{0}^{d\left(y_{2 k}, y_{2 k+1}\right)} \phi(t) d t \\
& +\gamma \int_{0}^{d\left(x_{2 k+1}, x_{2 k+1}\right)} \phi(t) d t+\delta \int_{0}^{d\left(x_{2 k+1}, x_{2 k+2}\right)} \phi(t) d t .
\end{aligned}
$$

Hence

$$
\int_{0}^{d\left(x_{2 k+1}, x_{2 k+2}\right)} \phi(t) d t \leq \frac{\alpha}{(1-\delta)} \int_{0}^{d\left(x_{2 k}, x_{2 k+1}\right)} \phi(t) d t+\frac{\beta}{(1-\delta)} \int_{0}^{d\left(y_{2 k}, y_{2 k+1}\right)} \phi(t) d t .
$$

Similarly, we have

$$
\begin{aligned}
\int_{0}^{d\left(y_{2 k+1}, y_{2 k+2}\right)} \phi(t) d t & =\int_{0}^{d\left(F\left(y_{2 k}, x_{2 k}\right), F\left(y_{2 k+1}, x_{2 k+1}\right)\right)} \phi(t) d t \\
& \leq \alpha \int_{0}^{d\left(y_{2 k}, y_{2 k+1}\right)} \phi(t) d t+\beta \int_{0}^{d\left(x_{2 k}, x_{2 k+1}\right)} \phi(t) d t \\
& +\gamma \int_{0}^{d\left(y_{2 k+1}, F\left(y_{2 k}, x_{2 k}\right)\right)} \phi(t) d t+\delta \int_{0}^{d\left(y_{2 k+1}, F\left(y_{2 k+1}, x_{2 k+1}\right)\right)} \phi(t) d t \\
& \leq \alpha \int_{0}^{d\left(y_{2 k}, y_{2 k+1}\right)} \phi(t) d t+\beta \int_{0}^{d\left(x_{2 k}, x_{2 k+1}\right)} \phi(t) d t \\
& +\gamma \int_{0}^{d\left(y_{2 k+1}, y_{2 k+1}\right)} \phi(t) d t+\delta \int_{0}^{d\left(y_{2 k+1}, y_{2 k+2}\right)} \phi(t) d t .
\end{aligned}
$$




\section{Hence}

$$
\int_{0}^{d\left(y_{2 k+1}, y_{2 k+2}\right)} \phi(t) d t \leq \frac{\alpha}{(1-\delta)} \int_{0}^{d\left(y_{2 k}, y_{2 k+1}\right)} \phi(t) d t+\frac{\beta}{(1-\delta} \int_{0}^{d\left(x_{2 k}, x_{2 k+1}\right)} \phi(t) d t .
$$

Adding (3) and (4) we get

$$
\begin{aligned}
\int_{0}^{d\left(x_{2 k+1}, x_{2 k+2}\right)} \phi(t) d t & +\int_{0}^{d\left(y_{2 k+1}, y_{2 k+2}\right)} \phi(t) d t \leq \frac{\alpha+\beta}{(1-\delta)} \int_{0}^{d\left(x_{2 k}, x_{2 k+1}\right)} \phi(t) d t \\
& +\frac{\alpha+\beta}{(1-\delta)} \int_{0}^{d\left(y_{2 k}, y_{2 k+1}\right)} \phi(t) d t \\
& \leq \frac{\alpha+\beta}{(1-\delta)}\left[\int_{0}^{d\left(x_{2 k}, x_{2 k+1}\right)} \phi(t) d t+\int_{0}^{d\left(y_{2 k}, y_{2 k+1}\right)} \phi(t) d t\right] \\
& =r\left[\int_{0}^{d\left(x_{2 k}, x_{2 k+1}\right)} \phi(t) d t+\int_{0}^{d\left(y_{2 k}, y_{2 k+1}\right)} \phi(t) d t\right],
\end{aligned}
$$

where $0<r=\frac{\alpha+\beta}{1-\delta}<1$.

Hence, from (5) we have

$$
\begin{aligned}
\int_{0}^{d\left(x_{2 k+2}, x_{2 k+3}\right)} \phi(t) d t & +\int_{0}^{d\left(y_{2 k+2}, y_{2 k+3}\right)} \phi(t) d t \\
& \leq r\left[\int_{0}^{d\left(x_{2 k+1}, x_{2 k+2}\right)} \phi(t) d t+\int_{0}^{d\left(y_{2 k+1}, y_{2 k+2}\right)} \phi(t) d t\right]
\end{aligned}
$$

Therefore

$$
\begin{aligned}
\int_{0}^{d\left(x_{n}, x_{n+1}\right)} \phi(t) d t+\int_{0}^{d\left(y_{n}, y_{n+1}\right)} \phi(t) d t & \leq r\left[\int_{0}^{d\left(x_{n-1}, x_{n}\right)} \phi(t) d t+\int_{0}^{d\left(y_{n-1}, y_{n}\right)} \phi(t) d t\right] \\
& \leq r^{2}\left[\int_{0}^{d\left(x_{n-2}, x_{n-1}\right)} \phi(t) d t+\int_{0}^{d\left(y_{n-2}, y_{n-1}\right)} \phi(t) d t\right] \\
& \ldots \ldots \ldots \\
& \leq r^{n}\left[\int_{0}^{d\left(x_{0}, x_{1}\right)} \phi(t) d t+\int_{0}^{d\left(y_{0}, y_{1}\right)} \phi(t) d t\right] .
\end{aligned}
$$

Now if we take

$$
\int_{0}^{d\left(x_{n}, x_{n+1}\right)} \phi(t) d t+\int_{0}^{d\left(y_{n}, y_{n+1}\right)} \phi(t) d t=\lambda_{n}
$$

then,

$$
\lambda_{n} \leq r \lambda_{n-1} \leq \cdots \leq r^{n} \lambda_{0} .
$$


Let $m>n$. Then using triangular inequality,

$$
\begin{array}{rl}
d\left(x_{m}, x_{n}\right) \leq \sum_{i=n}^{m-1} & d\left(x_{i}, x_{i+1}\right) \\
\int_{0}^{d\left(x_{n}, x_{m}\right)} \phi(t) d t+\int_{0}^{d\left(y_{n}, y_{m}\right)} \phi(t) d t & \leq \lambda_{m-1}+\lambda_{m-2}+\cdots+\lambda_{n} \\
& \leq\left(r^{m-1}+r^{m-2}+\cdots+r^{n}\right) \lambda_{0} \\
& =r^{n}\left(1+r+\cdots+r^{m-n-1}\right) \lambda_{0} \\
& \ldots \ldots \\
& \leq \frac{r^{n}}{1-r} \lambda_{0} .
\end{array}
$$

Consequently,

$$
\begin{aligned}
& \int_{0}^{d\left(x_{n}, x_{m}\right)} \phi(t) d t \leq \frac{r^{n}}{1-r} \lambda_{0} \\
& \int_{0}^{d\left(y_{n}, y_{m}\right)} \phi(t) d t \leq \frac{r^{n}}{1-r} \lambda_{0} .
\end{aligned}
$$

Let $0 \ll c$ be given. Choose a natural number $N$ such that $\frac{r^{n}}{1-r} \lambda_{0} \ll c$ for $n>N$.

Since for all $0 \ll \epsilon, 0 \ll \int_{0}^{\epsilon} \phi(t) d t$ so $d\left(x_{n}, x_{m}\right) \ll c$ and $d\left(y_{n}, y_{m}\right) \ll c$ for $m>n$. Therefore both the sequences $\left\{x_{n}\right\}$ and $\left\{y_{n}\right\}$ are Cauchy sequences.

From the completeness of $X$, there exist $x, y \in X$ such that

$$
\lim _{n \rightarrow \infty} x_{n}=x \text { and } \lim _{n \rightarrow \infty} y_{n}=y
$$

Now we prove that $F(x, y)=x$ and $F(y, x)=y$. Substituting $x_{2 k+1}=F(x, y)$ and $y_{2 k+1}=F(y, x)$ in (5), we obtain

$$
\begin{aligned}
\int_{0}^{d\left(F(x, y), x_{2 k+2}\right)} \phi(t) d t & +\int_{0}^{d\left(F(y, x), y_{2 k+2}\right)} \phi(t) d t \\
& \leq r\left[\int_{0}^{d\left(x_{2 k}, F(x, y)\right)} \phi(t) d t+\int_{0}^{d\left(y_{2 k}, F(y, x)\right)} \phi(t) d t\right] .
\end{aligned}
$$

Letting $k \longrightarrow \infty$ we obtain

$$
\begin{aligned}
& \int_{0}^{d(F(x, y), x)} \phi(t) d t+\int_{0}^{d(F(y, x), y)} \phi(t) d t \leq r\left[\int_{0}^{d(x, F(x, y))} \phi(t) d t+\int_{0}^{d(y, F(y, x))} \phi(t) d t\right], \\
& \Longrightarrow \quad(1-r)\left[\int_{0}^{d(x, F(x, y))} \phi(t) d t+\int_{0}^{d(y, F(y, x))} \phi(t) d t\right] \leq 0 .
\end{aligned}
$$


Since $0<r<1$ so $(1-r)>0$ therefore

$$
\int_{0}^{d(x, F(x, y))} \phi(t) d t+\int_{0}^{d(y, F(y, x))} \phi(t) d t \leq 0 .
$$

Which is a contradiction.

Therefore,

$$
\int_{0}^{d(x, F(x, y))} \phi(t) d t+\int_{0}^{d(y, F(y, x))} \phi(t) d t=0 .
$$

Since for all $0 \ll \epsilon, 0 \ll \int_{0}^{\epsilon} \phi(t) d t$ so we have

$\Longrightarrow \quad d(F(x, y), x)=0$ and $d(F(y, x), y)=0$,

so $\quad F(x, y)=x$ and $F(y, x)=y$.

In order to prove the uniqueness let $\left(x^{\prime}, y^{\prime}\right)$ be another point of $X \times X$ such that $x^{\prime}=F\left(x^{\prime}, y^{\prime}\right)$ and $y^{\prime}=F\left(y^{\prime}, x^{\prime}\right)$. Then

$$
\begin{aligned}
& \int_{0}^{d\left(x, x^{\prime}\right)} \phi(t) d t=\int_{0}^{d\left(F(x, y), F\left(x^{\prime}, y^{\prime}\right)\right)} \phi(t) d t \\
& \leq \alpha \int_{0}^{d\left(x, x^{\prime}\right)} \phi(t) d t+\beta \int_{0}^{d\left(y, y^{\prime}\right)} \phi(t) d t \\
&+\gamma \int_{0}^{d\left(x^{\prime}, F(x, y)\right)} \phi(t) d t+\delta \int_{0}^{d\left(x^{\prime}, F\left(x^{\prime}, y^{\prime}\right)\right)} \phi(t) d t \\
& \leq \alpha \int_{0}^{d\left(x, x^{\prime}\right)} \phi(t) d t+\beta \int_{0}^{d\left(y, y^{\prime}\right)} \phi(t) d t \\
&+\gamma \int_{0}^{d\left(x^{\prime}, x\right)} \phi(t) d t+\delta \int_{0}^{d\left(x^{\prime}, x^{\prime}\right)} \phi(t) d t . \\
& \Longrightarrow(1-\alpha-\gamma) \int_{0}^{d\left(x, x^{\prime}\right)} \phi(t) d t \leq \beta \int_{0}^{d\left(y, y^{\prime}\right)} \phi(t) d t . \\
& \Longrightarrow \int_{0}^{d\left(x, x^{\prime}\right)} \\
& \phi(t) d t \leq \frac{\beta}{(1-\alpha-\gamma)} \int_{0}^{d\left(y, y^{\prime}\right)} \phi(t) d t .
\end{aligned}
$$

Similarly, we can prove that

$$
\int_{0}^{d\left(y, y^{\prime}\right)} \phi(t) d t \leq \frac{\beta}{(1-\alpha-\gamma)} \int_{0}^{d\left(x, x^{\prime}\right)} \phi(t) d t .
$$

Adding (8) and (9) we get

$$
\begin{aligned}
& \int_{0}^{d\left(y, y^{\prime}\right)} \phi(t) d t+\int_{0}^{d\left(x, x^{\prime}\right)} \phi(t) d t \leq \frac{\beta}{(1-\alpha-\gamma)}\left[\int_{0}^{d\left(x, x^{\prime}\right)} \phi(t) d t+\int_{0}^{d\left(y, y^{\prime}\right)} \phi(t) d t\right] . \\
& \Longrightarrow\left(1-\frac{\beta}{1-\alpha-\gamma}\right)\left[\int_{0}^{d\left(y, y^{\prime}\right)} \phi(t) d t+\int_{0}^{d\left(x, x^{\prime}\right)} \phi(t) d t\right] \leq 0 .
\end{aligned}
$$


Since $0<\left(1-\frac{\beta}{1-\alpha-\gamma}\right)<1$ so

$$
\begin{array}{cl} 
& \int_{0}^{d\left(y, y^{\prime}\right)} \phi(t) d t+\int_{0}^{d\left(x, x^{\prime}\right)} \phi(t) d t \leq 0, \\
& \Longrightarrow \quad \int_{0}^{d\left(y, y^{\prime}\right)} \phi(t) d t+\int_{0}^{d\left(x, x^{\prime}\right)} \phi(t) d t=0, \\
\text { i.e } \quad & d\left(x, x^{\prime}\right)=0 \quad \text { and } \quad d\left(y, y^{\prime}\right)=0, \\
\text { i.e } \quad x=x^{\prime} \quad \text { and } \quad y=y^{\prime} .
\end{array}
$$

Now the proof is completed.

Corollary 8. In Theorem 7 if we take $\gamma=0$ and $\delta=0$.Then we have,

Let $(X, d)$ be a complete cone metric space with a cone $P$ having nonempty interior.

Let $F: X \times X \longrightarrow X$ satisfying

$$
\int_{0}^{d(F(x, y), F(u, v))} \phi(t) d t \leq \alpha \int_{0}^{d(x, u)} \phi(t) d t+\beta \int_{0}^{d(y, v)} \phi(t) d t
$$

for all $x, y, u, v \in X$, and $\alpha, \beta \in(0,1)$ such that $\alpha+\beta<1$ and $\phi(t): P \rightarrow P$ is a Lebesgue-integral mapping which is summable, nonnegative and such that for all $0 \ll \epsilon, 0 \ll \int_{0}^{\epsilon} \phi(t) d t$.

Then $F(x, y)$ has a unique coupled fixed point in $X \times X$.

Corollary 9. In Theorem 7 if we take $\delta=0$.Then we have,

Let $(X, d)$ be a complete cone metric space with a cone $P$ having nonempty interior. Let $F: X \times X \longrightarrow X$ satisfying

$$
\int_{0}^{d(F(x, y), F(u, v))} \phi(t) d t \leq \alpha \int_{0}^{d(x, u)} \phi(t) d t+\beta \int_{0}^{d(y, v)} \phi(t) d t+\gamma \int_{0}^{d(u, F(x, y))} \phi(t) d t,
$$

for all $x, y, u, v \in X$, and $\alpha, \beta, \gamma \in(0,1)$ such that $\alpha+\beta+\gamma<1$ and $\phi(t): P \rightarrow P$ is a Lebesgue-integral mapping which is summable, nonnegative and such that for all $0 \ll \epsilon, 0 \ll \int_{0}^{\epsilon} \phi(t) d t$.

Then $F(x, y)$ has a unique coupled fixed point in $X \times X$.

Corollary 10. In Theorem 7 if we take $\gamma=0$. Then we have,

Let $(X, d)$ be a complete cone metric space with a cone $P$ having nonempty interior.

Let $F: X \times X \longrightarrow X$ satisfying

$$
\int_{0}^{d(F(x, y), F(u, v))} \phi(t) d t \leq \alpha \int_{0}^{d(x, u)} \phi(t) d t+\beta \int_{0}^{d(y, v)} \phi(t) d t+\delta \int_{0}^{d(u, F(u, v))} \phi(t) d t,
$$

for all $x, y, u, v \in X$, and $\alpha, \beta, \delta \in(0,1)$ such that $\alpha+\beta+\delta<1$ and $\phi(t): P \rightarrow P$ is a Lebesgue-integral mapping which is summable, nonnegative and such that for all $0 \ll \epsilon, 0 \ll \int_{0}^{\epsilon} \phi(t) d t$.

Then $F(x, y)$ has a unique coupled fixed point in $X \times X$. 


\section{EXAMPLE}

Let $E=\mathbb{R}$ and $P=\{x \in E: x \geq 0\}$. Let $X=[0,2]$ and define a mapping $d: X \times X \rightarrow X$ by $d(x, y)=|x-y|$. Then $(X, d)$ is a complete cone metric space. Define $F: X \times X \rightarrow X$ by $F(x, y)=\frac{x+y}{2}$ for all $x, y \in X$. Define $\phi(t): P \rightarrow P$ by $\phi(t)=t$ for all $t \in P$.

Then the condition of the theorem 7 is satisfied.

Here it is seen that $\left(\frac{1}{2}, \frac{1}{2}\right)$ is the unique coupled fixed point of $F(x, y)$.

\section{REFERENCES}

[1] L. G. Huang, X. Zhang, Cone metric spaces and fixed point theorems of contractive mappings, J. Math. Anal. Appl., 332 (2007), 1468-1476.

[2] T. G. Bhaskar, V. Lakshmikantham, Fixed point theorems in partially ordered metric spaces and applications, Nonlinear Anal., 65 (2006), no. 7, 1379-1393.

[3] V. Lakshmikantham, L. Ciric, Coupled fixed point theorems for nonlinear contractions in partially ordered metric spaces, Nonlinear Anal., 70 (2009), 4341-4349.

[4] V. L. Nguyen, X. T. Nguyen, Coupled fixed points in partially ordered metric spaces and application, Nonlinear Anal., 74 (2011), 983-992.

[5] E. Karapinar, Couple fixed point theorems for nonlinear contractions in cone metric spaces, Comput. Math. Appl., 59 (2010), 3656-3668.

[6] B. S. Choudhury, N. Metiya, The point of coincidence and common fixed point for a pair of mappings in cone metric spaces, Comput. Math. Appl., 60 (2010), 1686-1695.

[7] S. Bhaumik, S. K. Tiwari, Generalized common coupled fixed point theorem of nonlinear contractive mapping in cone metric space, International Journal of Pure and Applied Mathematics, 116 (2017), No. 4, 1127-1137.

[8] A. Branciari, A fixed point theorem of Banach-Caccioppoli type on a class of generalized metric spaces, Publ. Math. Debrecen, 57 (2000), 31-37. .

[9] B. S. Choudhury, P. Maity, Coupled fixed point results in generalized metric spaces, Mathematical and Computer Modelling, 54 (2011), no. 1-2, 73-79.

[10] Z. Kadelburg, S. Radenovic and B. Rosic, Strict contractive conditions and common fixed point theorems in cone metric spaces, Fixed Point Theory and Applications, vol. 2009 (Article ID 173838, 14 pp.), doi:10.1155/2009/173838.

[11] D. Ilic, V. Rakocevic, Common fixed points for maps on cone metric space, Journal of Mathematical Analysis and Applications, 341 (2008), 876-882. 
[12] A. Aliouche, A common fixed point theorem for weakly compatible mappings in symmetric spaces satisfying a contractive condition of integral type. Journal of Mathematical Analysis and Applications, 322 (2006), no. 2, 796-802.

[13] A. Branciari, A fixed point theorem for mappings satisfying a general contractive condition of integral type, Int. J. Math. Math. Sci., 29 (2002), no. 9, 531-536.

[14] A. Djoudi, A. Aliouche, Common fixed point theorems of Gregus type for weakly compatible mappings satisfying contractive conditions of integral type, J. Math. Anal. Appl., 329 (2007), 31-45.

[15] B. E. Rhoades, Two fixed point theorems for mappings satisfying a general contractive condition of integral type, Int. J. Math. Math. Sci., 63 (2003), 4007-4013.

[16] P. Vijayaraju, B. E. Rhoades, and R. Mohanraj, A fixed point theorem for a pair of maps satisfying a general contractive condition of integral type, Int. J. Math. Math. Sci., 15 (2005), 2359-2364. 